\title{
Veterinary and sanitary examination of yak slaughter products in case of invasive diseases in the conditions of the Primorsky Territory
}

\author{
Dmitrii Zamaratskii ${ }^{1}$, Margarita Simakova ${ }^{1}$, Guli Koltun $^{1}$, Viktoriia Podvalova ${ }^{1}$ \\ ${ }^{1}$ Primorskaya State Academy of Agriculture, pr. Blyukhera, 44, Ussuriysk, Primorsky Territory, \\ 692510, Russia
}

\begin{abstract}
Primorsky Territory is a platform for the intensification of the livestock industry in order to provide the population with high-quality meat products. However, the introduction of new species of animals (yaks, camels, highly productive breeds of cattle) to the territory of the region contributes to the emergence and spread of invasions among them. Fascioliasis, dictyocaulosis, piroplasmidosis are natural focal parasitic diseases of animals that are widespread in the Primorsky Territory. They are detected almost every day during the veterinary and sanitary examination of animal slaughter products. Despite the absence of a threat of infection with these diseases for humans through meat and by-products, there remains a risk of deterioration in the quality of such products and the development of defects in meat raw materials, which leads to the impossibility of further sale and consumption. Yaks were brought to the Primorsky Territory in 2010 as an experiment, and in 2015, farmers actively used these animals as beef cattle, since their simplicity in keeping significantly reduced the cost of the meat production process. The conditions of keeping yaks in the Primorsky Territory contributed to their infection with parasitic diseases, the extent of the invasion of which reached $60 \%$ and more.
\end{abstract}

\section{Introduction}

Yak - (lat. Bos mutus) - artiodactyl mammal from the genus of true bulls of the Bovidae family. In the Ussuriysky urban district of the Primorsky Territory, yaks for breeding were brought in 2015 from Buryatia to the Tolochka V.V. peasant farm.

Yaks, having high adaptive qualities to difficult climatic conditions, are able to use pastures that are inaccessible to other species of animals, which, at minimal cost, allows obtaining high quality products [4]. Yak meat is ecologically clean, dietary (100 grams of yak meat contains about $110 \mathrm{kcal}$ ), complete in composition and the richest in hemoglobin content [10]. Yaks are generally not susceptible to widespread animal diseases. These factors have a beneficial effect on the body of the yak and, as a result, on their offal and meat. The uniqueness of this product lies in the fact that it contains a large amount of easily digestible iron. Its regular use helps to strengthen the musculoskeletal system, reduces the 
permeability of blood vessels, stimulates the processes of metabolism and hematopoiesis, has an antioxidant, anti-inflammatory, and tonic effect $[8,9]$.

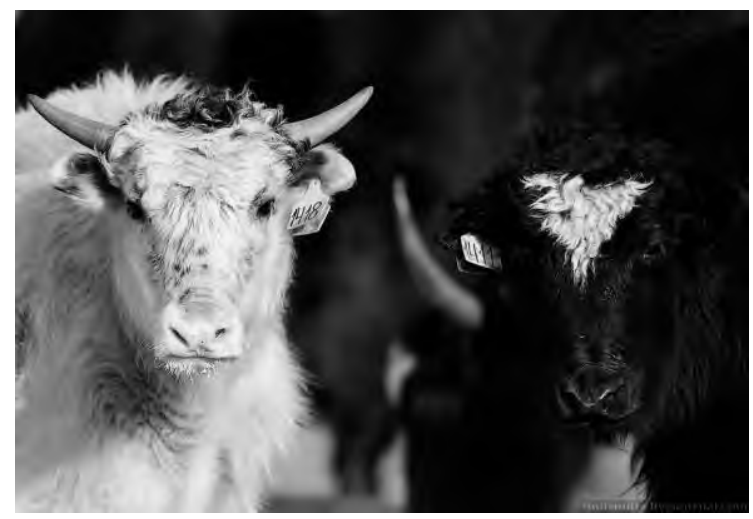

Fig. 1. Yaks of the Oka breed in the Tolochka V.V. peasant farm.

However, yaks are susceptible to parasitic diseases. In the works of A.M. Bittirov (1992, 1999), K.K. Umarov (1994), Oshkhunov A.K. (1999), there are brief reports on the presence of various types of helminths (trematodes, larval cestodes and nematodes) in the slaughter products of yaks. According to these authors, the invasion of yaks in the conditions of Tyzyl, Khaznidon, Sukan, Bezengi, Shiki, Bashil pastures (KabardinoBalkarian Republic) with fascioliasis is $64.8 \pm 10.2 \%$, with dicrocoeliasis - $87.3 \pm 11.5 \%$, larval echinococcosis - 47.6+5.4\%, cysticercus bovis - 8.8+3.6\%, moniesiasis (calves) $35.1+8.3 \%$, dictyocaulosis (calves) $-46.4+10.2 \%$, strongyloidiasis of the gastrointestinal tract $-100 \%$ with weak, medium and strong intensity of helminths $[3,4,5]$. There are reports of M.Sh. Akbaev and A.K. Oshkhunov (1994) that helminthiases account for $18-32 \%$ of yaks' mortality in different years, the joints of which are found in the highlands in the form of helminthic faunal complexes and cause a severe course of invasions [6,7]. With the above-mentioned helminthiasis of yaks, the increase in body weight is reduced by $25-40 \%$. It was found that during the growing season, an average of $28.6 \mathrm{~kg}$ of body gain was not received from the infested three-year-old yaks $[1,2]$.

Based on the above and taking into account the fact that yaks for industrial breeding were brought to the Primorsky Territory for the first time, the purpose of the study was to study the incidence of invasive diseases in yaks in the Primorsky Territory. In this regard, it was necessary to complete a number of tasks:

1. To study the data of the veterinary reporting of the RSBI "Ussuriysk Veterinary Station for Combating Animal Diseases" on the registered cases of detection of parasitosis in the slaughter products of yaks.

2. Conduct control slaughter of yaks and veterinary and sanitary examination of yak meat in the laboratory of veterinary and sanitary examination of FSBEI HE Primorskaya State Academy of Agriculture.

3. Analyze the data obtained and give an opinion on the quality of yak meat, including in cases of detection of invasive animal diseases.

\section{Materials and methods}

The work was carried out in the laboratory of veterinary and sanitary examination of the RSBI "Ussuriysk Veterinary Station for Combating Animal Diseases" and the laboratory of veterinary and sanitary examination of the Federal State Budgetary Educational Institution of Higher Education Primorskaya State Academy of Agriculture. The object of the study 
was yak carcasses provided by farmers for the veterinary and sanitary examination of slaughter products.

To confirm the diagnoses and further use of yak meat and offal, a veterinary and sanitary examination was carried out in accordance with the "Rules for veterinary examination of slaughtered animals and veterinary and sanitary examination of meat and meat products". Also, the internal organs were examined separately for the presence of parasites using the technique of incomplete parasitological dissection.

For the statistical analysis of data on the incidence of invasive diseases in yaks, the veterinary accounting and reporting documentation of the RSBI "Ussuriysk Veterinary Station for Combating Animal Diseases" was used, containing statistical data on the postmortem detection of animal parasitic diseases in the period 2017-2019.

\section{Research results}

Before the beginning of the experimental part, we studied the veterinary accounting and reporting documentation containing statistical data on the post-mortem detection of animal parasitic diseases in the period 2017-2019.

When assessing the epizootic status of the territory of the Ussuriysk urban district for parasitic diseases, we used the data of reports of the 5-vet form.

According to reports on the results of veterinary examination of slaughtered animals and veterinary and sanitary examination of meat in the period from 2017 to 2019, parasitic pathology in yaks is represented mainly by three nosological units. In 2017, 21 cases of invasive diseases (dictyocaulosis, fascioliasis, piroplasmosis) were identified. In 2018, 33 cases (dictyocaulosis, piroplasmosis, fascioliasis), and in 2019, 13 cases (dictyocaulosis, piroplasmosis, fascioliasis) were registered.

The results of post-mortem diagnostics of parasitic diseases of yaks are shown in Fig. 2.

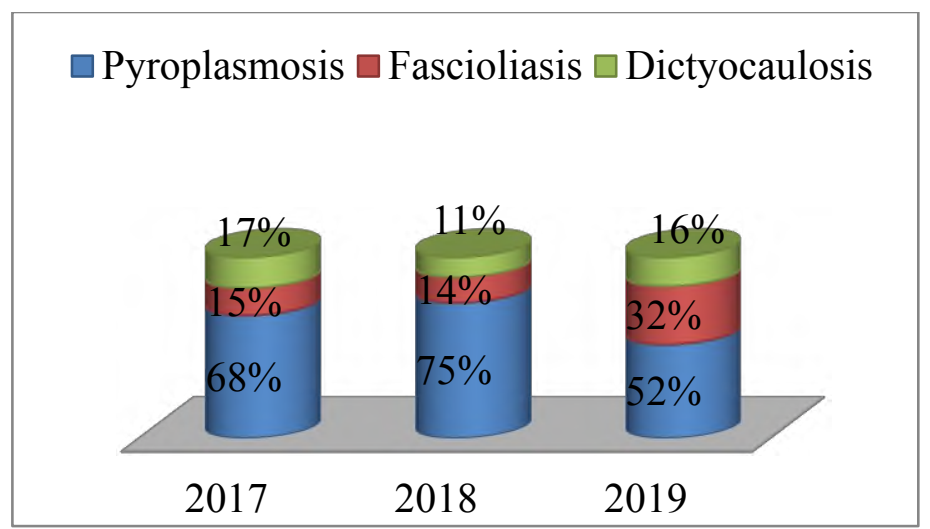

Fig. 2. Nosological profile of parasitic diseases of yaks.

The next stage of our research was the veterinary and sanitary examination of the carcasses and internal organs of yaks.

Post-slaughter veterinary and sanitary examination of carcasses and internal organs of yaks was carried out in accordance with the "Rules for Veterinary Examination of Slaughter Animals and Veterinary and Sanitary Examination of Meat and Meat Products" (1988), according to the generally accepted method, as in cattle.

During the examination of the head, attention was paid to the mucous membranes of the lips, tongue, mouth and eyes, lymph nodes of the head. The chewing muscles were examined according to the generally accepted method. For this, two longitudinal incisions 
of the external and one incision of the internal masticatory muscles were made in order to exclude cysticercus bovis (finnosis), the presence of which was not detected.

When examining the internal organs, attention was paid to the appearance, color, size, consistency, blood filling, the state of the capsule and parenchyma of the organ. The spleen was examined externally and in section. It is reddish-brown in color, elongated-oval with sharp edges, dense consistency, the pulp is dark cherry color in the cut. The lungs were examined from the outside and all lobes of the lung were palpated. Normally, the lungs are pink, conical, with sharp edges. Unlike the lungs of other ruminant species, the left lung of the yak consists of two lobes, where one of the lobes is the merged cranial and cardiac lobes, while the lungs of the first ones are represented by three lobes - the cranial, cardiac and caudal lobes. The heart of a yak is cone-shaped; it was examined after opening the pericardium, no peculiarities were noted. Normally, the liver has a dark brown color, dense texture, sharp edges. The gallbladder is moderately filled with bile. Portal lymph nodes are bean-shaped and bifurcated-flattened. The content of the bile ducts was examined by several longitudinal incisions of the liver tissue, and no changes were found. When examining the kidneys, it was found that the kidneys of a yak are bean-shaped, brown-red, bumpy, the capsule can be easily removed. In the cut, the structure of the organ is well expressed. By the type of structure, the yak kidney belongs to the grooved multi-papillary. The stomach was examined from the outside without opening it for examination. Intestine from the side of the serous membrane, followed by incision of the mesenteric lymph nodes. The udder was palpated, consistency, color and odor were determined, and two deep cuts were made.

When examining the carcasses, attention was paid to the appearance, fatness, degree of exsanguination, color, odor of muscle tissue and fat, condition of subcutaneous tissue, pleura, peritoneum and joints, which made it possible to determine the veterinary and sanitary quality of meat.

During the veterinary and sanitary examination of carcasses and internal organs of yaks, special attention was paid to the superficial lymph nodes. In terms of morphological structure, the lymph nodes of yaks are integral undivided formations, similar to the lymph nodes of cattle. The shape of the lymph nodes is round or oval, bean-shaped, there are flattened-bifurcated (accessory lobes of the right lung and one of the portal ones), the surface has a light gray color, the size can be different. In the cut, the cortical (outer) and cerebral (inner) zones are hardly distinguishable. The cortical is lighter in color than the cerebral. Of the lymph nodes of yaks, the most accessible and convenient for research are in the head area: submandibular, parotid, retropharyngeal middle and lateral; in the liver bronchial left and right, anterior, middle and caudal mediastinal, as well as the lymph node of the accessory lobe of the right lung; on the carcass - superficial and deep cervical, axillary, thoracic anterior, intercostal, lumbar, iliac, knee folds, sacral, inguinal and popliteal lymph nodes.

When conducting a veterinary and sanitary examination of the slaughter products of yaks, it was found that in the period from 2017-2019, the invasion of the latter was: piroplasmosis - $19.5 \%$; fascioliasis - $61 \%$; dictyocaulosis - $44 \%$.

In the liver affected by fascioliasis (Fasciola hepatica), pathomorphological changes characterizing the presence of parasites were noted. On examination, spotted and punctate hemorrhages were recorded under the capsule and in the parenchyma of the organ. There was a thickening of the bile ducts in the form of yellow cords of varying thickness on the portal surface of the liver. When cut, dirty brown or dirty brick bile with an admixture of live fascioli and scraps of decayed parasites flowed out of the bile ducts. The lymph nodes of the liver are enlarged, juicy and dark brown in color in the cut.

In some yaks, depending on the growth and development of the fascioli, the liver was greatly enlarged in size, brown-red in color, the edges of the lobes were tense, and the 
surface of the organ was rough. On the diaphragmatic side of the liver, there were holes "drilling paths". The holes in the liver capsule were filled with blood clots, from which the pointed tips of the young fascioli that had not yet emerged protruded. When pressing on the surrounding tissue, the parasites were released from the holes; in the cut, the entire parenchyma of the liver was penetrated by thin blood passages.

Examination of the lungs with lesions by dictyocaulosis also revealed pathological changes. The lungs were of dense consistency; whitish-gray nodules of various sizes protruding above the surface of the organ were noted on the serous membrane. The mucous membrane of the bronchi had hemorrhages, the walls are thickened, hard to the touch, the Dictyocaulus filarial nematodes were found in the small bronchi of the lungs.

An objective conclusion about the degree of freshness of yak meat was made on the basis of a complex of indicators of organoleptic evaluation, microscopy of imprint smears, determination of the products of primary protein breakdown in broth (reaction with copper sulfate).

During storage, the meat was subjected to a comprehensive study every day, as a result of which the organoleptic, chemical and microscopic indicators of fresh, doubtful fresh and tainted meat were determined. Yak meat normally has a specific smell, from dark red to cherry color, it is moist in the cut, but does not leave wet spots on the filter paper, the fat is orange-yellow in color. It was found by cooking tests that the broth is transparent and has a specific aromatic smell. The abovementioned organoleptic indicators of the freshness of yak meat were preserved during the first day. Microscopic studies in the first 3 days showed the absence or detection of single microorganisms, the absence of traces of decay of muscle fibers. The products of primary protein degradation were not detected in the broth in the first 3 days, the broth remained transparent when copper sulfate was added.

Significant changes occur with the meat of animals infected with nematodes on the 4th day of storage, an unpleasant (musty or slightly acidic) smell appears, the color, consistency and moisture of the muscles in the cut change. The meat acquires an uneven color from dark red to brown, the consistency of the meat weakens, the muscles in the cut are moister, they leave a moist spot on the filter paper. At the same time, microscopic and chemical parameters of meat change. Microscopy of imprint smears revealed traces of decay of muscle fibers and up to 25 microorganisms in the field of view.

Decomposition products of primary proteins are detected in the broth. When a $5 \%$ solution of copper sulfate is added, the broth becomes turbid. According to the above indicators, yak meat belongs to the category of questionable freshness.

Deeper changes in yak meat are observed on the 5 th day of storage. The meat acquires a dull gray color from the surface, sticky, smell from sour to putrid. A large number ( 25 or more) of microorganisms are found in imprint smears. Significant breakdown of muscle fibers is observed.

When a $5 \%$ solution of copper sulfate is added to the broth, a jelly-like precipitate falls out (positive result). Organoleptic, chemical and microscopic indicators of freshness of yak meat on the 5th day of storage correspond to those of tainted meat.

The meat of yaks heavily infested with trematodes is fresh in the first 24 hours after slaughter. According to the organoleptic characteristics, the meat is dark red to cherry color, the consistency is elastic, when pressed with a finger, a pit is formed, which gradually leveled out within 1-2 minutes, it is wet in the cut, but does not leave a wet spot on the filter paper, the smell is specific, the color of the meat is uniform. The broth is transparent, the smell is pleasant, specific. During microscopy of imprint smears, up to 10 20 staphylococci and streptococci are found in the field of view; the broth is clear, does not contain sediment, which meets the requirements of the "Rules for Veterinary Examination of Slaughter Animals and Veterinary and Sanitary Examination of Meat and Meat Products". 
On the 2-3rd day of storage, the meat acquires signs of doubtful freshness: it has dark cherry color in the cut, it is slightly dull from the surface, the smell is sour, it is moist in the cut, it leaves a damp spot on the filter paper from dark red to brown color, the consistency is inelastic. The broth is slightly turbid, has a sourish taste. During microscopy of imprint smears, more than 30 microorganisms and traces of decay of muscle fibers are found in the field of view of the microscope. The broth is turbid, the content of volatile fatty acids in various meat samples varies in the range of $3.3-7.9 \mathrm{mg}$, which allows attributing it to doubtful freshness. It is recommended to use meat of doubtful freshness only in the production of cooked sausages. On the 5-7th day of storage, the processes of putrefactive decay of proteins, seeding with microorganisms, decay of muscle fibers, changes in organoleptic and physicochemical parameters characteristic of tainted meat prevail in the meat of yaks infested with trematodes.

\section{Conclusion}

Thus, we have established that, despite the absence of signs of post-slaughter changes in the muscle tissue of yaks infected with helminths in the first day after the slaughter, changes characteristic of a stale product appear later when storing chilled yak meat. Therefore, such meat is not subject to sale, since it is unsafe for human health.

\section{References}

1. A.A. Abdykerimov, Growth, development and meat quality of young yaks in different breeding methods, Abstract of thesis for a degree of PhD in Agriculture. Frunze, 14 (1971)

2. A.M. Bittirov, Pathological changes in the liver with fascioliasis in cattle, Journal of veterinary medicine, 2, 47-50 (1996)

3. A.M. Bittirov, Impact of Fasciola hepatica symbionts on animals // Journal of veterinary medicin. Stavropol, 2, 50-52. (1996)

4. G.V. Ivanchuk, D.V. Zamaratsky, A.A. Verzun Topical issues of the development of food production: technology, quality, ecology, equipment, management and marketing. Proceedings of the III All-Russian scientific and practical conference, 50-56 (2019)

5. Parasite fauna of yaks in the conditions of Kabardino-Balkaria and measures to combat the main pathogens: Abstract of thesis for a degree of PhD in Biology: 03.00.19/ Moscow State Academy of Veterinary Medicine and Biotechnology named after K.I. Scriabin, 18 (1999)

6. R. J. Christopherson, R. J. Hudson Nortehm animal agriculture, Agn. Forestry Bull., 1.2, 3-5 (1970)

7. Cheng Peilieu. Jak (types) Livestock breeds of China. FAO Animal production and health paper ,96-108 (1984)

8. H. Epstein Jak and chauri, Wold anim Rev, 9, 8-12 (1974)

9. J.C. Jain, R.S. Jabova Jield and composition of milk of Himacheli yak hybrid hill cow, Jndianj anim. Sc, 55, 223-224 (1985)

10. J.A. Libby Meat higien, 4 ed. Philadelphia, Lea and Febiger 280 (1975) 